\title{
Has the Local Area Network (LAN) the Same Characteristics With the Particle/field Entity? Recovering the Schwarzschild Metric in a Discrete and Flat Spacetime, Using a Diffusion-driven Impurity Model Produced by Partile/LAN Entities.
}

Aris Chatzichristos ( $\square$ arishadj@gmail.com )

University of British Columbia

\section{Research Article}

Keywords: Local Area Network (LAN), particle/field entity, Schwarzschild metric, impurity model, Átmiton theory

Posted Date: April 19th, 2021

DOI: https://doi.org/10.21203/rs.3.rs-404695/v1

License: (c) (1) This work is licensed under a Creative Commons Attribution 4.0 International License. Read Full License 


\title{
Has the Local Area Network (LAN) the same characteristics with the particle/field entity? Recovering the Schwarzschild metric in a discrete and flat spacetime, using a diffusion-driven impurity model produced by partile/LAN entities.
}

\author{
A. Chatzichristos ${ }^{1,2, *}$ \\ ${ }^{1}$ Department of Physics and Astronomy, University of British Columbia, Vancouver, BC V6T 1Z1, Canada \\ ${ }^{2}$ Stewart Blusson Quantum Matter Institute, University of British Columbia, Vancouver, BC V6T 1Z4, Canada \\ *aris.chatzichristos@alumni.ubc.ca
}

\begin{abstract}
A local area network (LAN) consists typically of several telecommunication nodes, all of which share a "public IP" address and a single point of contact with the Internet, namely the router. For an outside observer, the whole LAN with all its nodes would look point-like (having one, shared, public IP address and a single connection with the Internet), but in its interactions it would appear field-like, as a LAN of several nodes, each with its own "private IP" address, has an internal structure and interacts with the outside world differently than a single node would. The above particle/LAN analogy will be used as a basis of Átmiton theory, according to which, elementary particles are made of LANs consisting of a type of telecommunication node - called átmiton - and space is a 3D network of átmita with the simple cubic topology.

In this work, it is demonstrated that connecting a particle/LAN entity on the lattice of space distorts its local topology, effectively introducing a crystal defect to the lattice of space. This defect increases the distance between points lying at opposite sides of it. The internal interactions of any massive object should be producing constantly copious numbers of such space-defects, which diffuse away from their source, by means of a random walk.

Here it is suggested that general relativity's notion of spacetime's curvature is equivalent to the aforementioned diffusion-driven impurity model in the flat, discrete lattice of space of Átmiton theory. Their equivalency is demonstrated for the specific case of a spherical mass, around which the Schwarzschild metric is shown to be valid.
\end{abstract}

\section{Introduction}

In the heart of quantum mechanics (QM) lies a series of "paradoxes", which are fully described mathematically and surveyed experimentally, but nonetheless contradict common sense. These include (amongst others) the particle/field duality, the spin being a type of angular momentum, except that point-like particles cannot rotate physically, or the fact that in a double-slit experiment an interference pattern arises even when the electrons are emitted one at the time. This is not to say that electrons do not have both particle and wave characteristics, but rather to stress that these effects contradict common sense in the context of the current formalism of QM. Many interpretations of QM exist, such as the Copenhagen ${ }^{1}$, De Broglie-Bohm ${ }^{2}$ and the Many Worlds ${ }^{3}$, but a comprehensive, intuitive picture of these phenomena is to some extend still absent. It is thus possible that to understand the aforementioned paradoxes in an intuitively comprehensive manner, one might have to move away from conventional pictures such as particles or fields whatsoever and find another mental frame that is better suited for the task at hand. Indeed, during the past few decades, several approaches tried to do just that, such as String/M-theory ${ }^{4}$, Loop Quantum Gravity ${ }^{5}$ and others. This study builds the case for another candidate replacement of the particle/field entity, namely the local area network.

Local area network (LAN) is a communication network of nodes (typically computers, smartphones, etc) that are interconnected through a network topology (meaning the map of the nodes' connections) and are able to exchange information. Information exchange between two nodes in the same LAN makes use of a single identification number for each node, called the private IP address (IP stands for "Internet Protocol"), assigned to them by a special node, called the router. If a node in the LAN wants to connect with a node outside the local network (e.g., with a computer in another LAN), it can do so through a router that is connected with the Internet, which is a network of networks.

To communicate through the Internet, all nodes in the same LAN share the same, single identification number, called the public IP address, and send messages to the outside world with the router acting as the sole gateway. In an abstract space 
depicting the topology of the interconnections of all nodes on the Internet based on their (public) IP addresses, a LAN would look point-like, in the sense that all of its nodes have the same public IP address and the LAN has a single point of contact with the Internet (see Fig. 1a). On the other hand, the fact that the LAN has actually some internal structure makes a large difference in regard to the way it interacts with the rest of the world, as a LAN consisting of a single node would create, process and demand data in a completely different way than a LAN consisting of hundreds of telecommunication nodes interconnected with a certain network topology (compare LAN1 and LAN2 in Fig. 1b). In other words, a LAN can be thought as having both particle and field characteristics, in analogy to an elementary particle in quantum (field) theory.

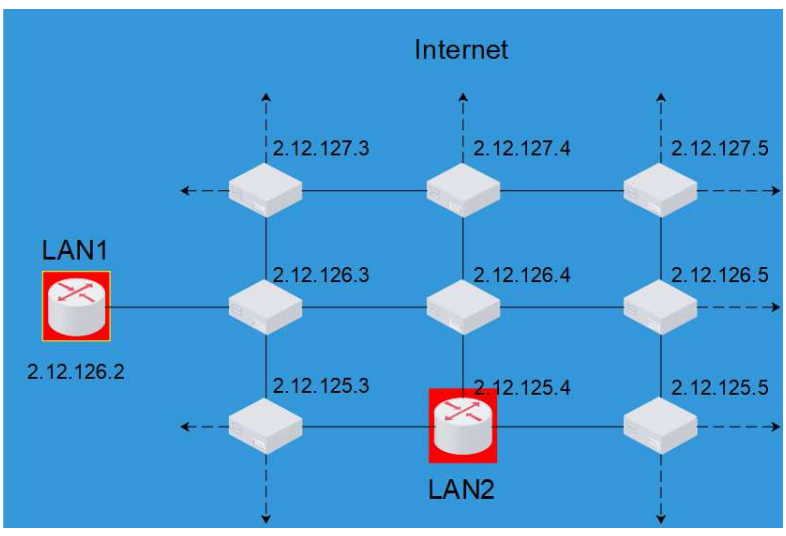

(a)

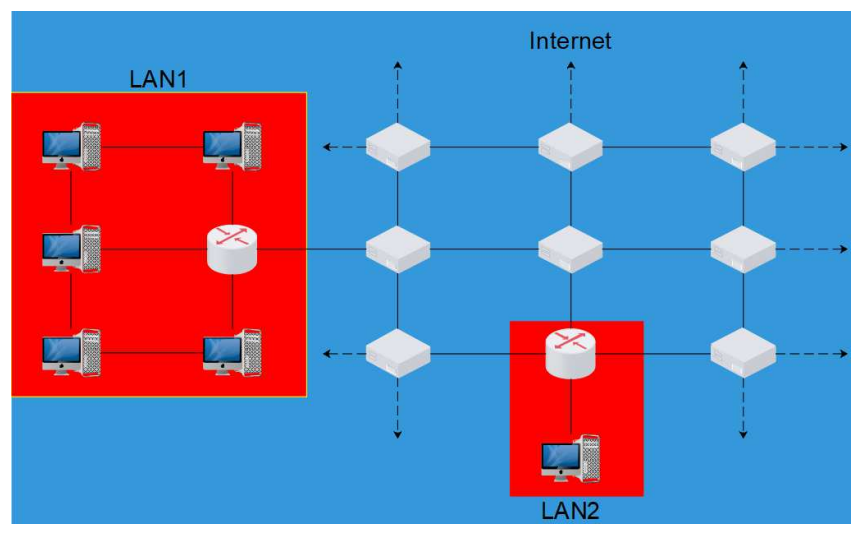

(b)

Figure 1. Image of two simple LANs (in red), each with a different number of internal nodes (computers), connected to the Internet (in blue) through a router. (a) Map of the public IP addresses. In this view, each LAN seems point-like, as it has one direct connection to the Internet and a single public IP address. (b) The map of all connections between nodes on the same network. Note that in telecommunication networks, the IP addresses of neighboring nodes are generally independent of each other, not in a series as depicted here.

The fundamental premise of Átmiton theory is that all particles are systems of LANs, each type having a certain internal configuration. On the one hand, this analogy provides a mental picture of how an expansive entity - like a field or a wave can appear point-like from outside. On the other hand, accepting the aforementioned premise, immediately leads to several important notions:

1) What we perceive as Space would correspond to the Internet: A network of telecommunication nodes on which LANs are connected. The position of an elementary particle would correspond to where the LAN's router is connected with the Internet/Space (as well as its public IP address). Obviously, space and time are discrete in this setting.

2) Since all nodes inside the LAN are not directly connected with the Internet/Space, they would have to send their signals to the outside world through the router, which would translate them in a form that can propagate in Space (i.e., through a process similar to translating the private IPs to a public IP address, organize how the signal will be transported on the web, etc). Such a translation process could be relevant to the long-range forces (Gravity, EM). But if two LANs approach each other, it is conceivable that they can connect directly, bypassing the Internet/Space, e.g., by forming a unified LAN. Henceforth, the signals exchanged between nodes belonging to the former two LANs won't have to be translated by the routers, so these untranslated signals that use directly the (equivalent of the) private IP addresses would be relevant to the short-range forces (Weak and Strong) and would probably exhibit very different characteristics.

3) It should be possible to embed a new LAN inside the initial LAN. We will call the initial LAN a substrate. The substrate LAN is external, in the sense that has a direct connection with the Internet/Space, whereas the new LAN is internal, as it has no direct connections with Space. The internal LAN will seem point-like to an observer at some node of the substrate LAN, while an observer outside both LANs (i.e., on the Internet/Space) would deem the whole structure as being point-like. If we now allow the internal LAN to be hopping around the substrate by gradually shifting its router's connection point from one node of the substrate to the next in a circular manner with a characteristic period (Fig. 2), then this motion can be thought (after appropriate definitions) to be carrying spin (i.e., intrinsic angular momentum). For an observer on the Internet/Space this spin would have no physical meaning, as they would perceive the whole structure of the two nested LANs as a single point (see LAN1 of Fig. 1a), incapable of having internal motions/rotations, but at the same time, this spin would manifest itself through the interactions of the nested system with other LANs. For instance, the time it takes for a signal coming from outside the nested LANs' system to arrive to a specific node of the internal LAN, would depend on the distance between the routers of the internal and the substrate LANs, which would be fluctuating due to this periodic motion. Hence, if there is a correlation 
between the strength of the interactions between two such nested systems and the time-lag of their exchanged signals, then this strength could be fluctuating with time, due to the rotation of the internal LAN on its substrate. Note that this rotation is a motion on a field (i.e., the substrate) and not in Space.

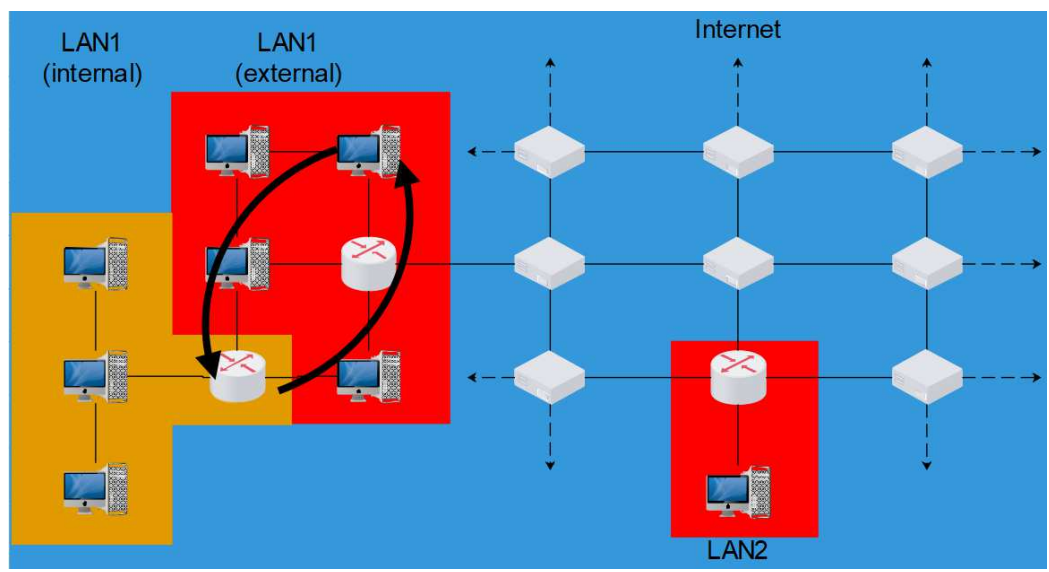

Figure 2. Image of an internal LAN, placed inside LAN1, with its router's position on the (external) LAN1 shifting counter-clockwise. Under appropriate definitions, such a motion can be associated with intrinsic angular momentum, even though the whole LAN1 seems still point-like, from the point of view of an outside observer (see Fig. 1a).

4) Another important consideration for this model is the definition of time. If we allow all nodes to be switching positions with one of their nearest neighbors in every LAN once per unit time (Fig. 2), then this hop rate would define the highest possible speed (i.e., the speed of light) and each such "turn" the quantum of time, but it would impose no upper speed boundary for information propagation. This instantaneous information exchange would allow for two LANs to share a virtual connection over large distances, possibly explaining how two far-away particles can form an Einstein-Podolsky-Rosen (EPR) connection ${ }^{6,7}$. Although this seems at a first glance to be at odds with Relativity, it is not, as this stream of information is not what observers can access directly.

To further elucidate how information propagation would work in this model, consider a bishop on a chessboard. From his point of view, time moves on discrete steps (each one being a player's turn), with the topology of the chessboard evolving after each of them. Under such a definition of time, the bishop (the equivalent of information in this analogy) can move "instantaneously" from one side of the board to the other, provided that each square on his way is open. So although information (the bishop) can move by multiple cells per unit time (in a players turn), allowing for the EPR effect, at the same time Relativity stems from the fact that the chessboard structure changes only once per unit time, in all players' frames of reference.

From the above considerations, it looks like the LAN telecommunication structure has (qualitatively) the equivalent of certain quantum properties. Here, we will follow this analogy and demand that all particles are networks of communication nodes (called "átmita") and exchange information through Space in an instantaneous but causal manner (just like the motion of the bishop on the board). The Space we perceive would be the equivalent of the public IP address topology of Fig. 1a and the time we perceive would be the multiples of the elementary steps with which the network topology changes (which we'll call a gyros, i.e., a "turn"). The advantage of this construct is that it requires only one species of "nodes" and one communication protocol.

The focus of this publication is the issue of placing such a LAN of átmita in Space. This action is not straightforward, as Space has (in the context of Átmiton theory) the topology of a perfect simple-cubic crystal (i.e., with no holes and defects) and only integer addresses are valid, so we can place the LAN's router at neither a substitutional nor at an interstitial position. Placing a particle/LAN entity in Space will be shown to create an effective curvature, as each particle acts as an "impurity" in Space (Sect. 3), much like a crystalline defect in a condensed matter system.

Following this result, it will be argued that massive objects generate great amounts of such space-impurities, due to all their internal interactions. These impurities then diffuse in all directions, driven by a concentration gradient. This mechanism will be suggested to be equivalent to the mass-generated curvature of general relativity $(\mathrm{GR})^{8}$. Indeed, to demonstrate the equivalency of the impurity-diffusion effect and GR's curvature notion, in Sect. 4 the Schwarzschild metric ${ }^{9}$ is reproduced, in the discrete and inherently flat spacetime of Átmiton theory. 


\section{Formulation of Átmiton theory}

Let an entity with no internal structure, called átmiton, that can form a network with other átmita by exchanging information that establish connections. In this Section we will gradually explore some aspects of the átmiton and its interactions, such as what a connection is, how information is propagating, what is the topology of the network and what sort of signals are exchanged between átmita.

The fundamentals of Átmiton theory consist of the general traits defining an átmiton, as well as a communication protocol that permits them to interact and form compound systems. It is the only ingredient of this theory, in the sense that everything else (i.e., empty space, particles/fields, etc) are considered to be systems of interacting átmita, with their network topology being what gives rise to the different characteristics of these systems.

In its essence, an átmiton resembles a telecommunication node, or a simple computer on a network. It interacts with the other átmita on the network by sending signals that propagate through the network in an instantaneous but causal manner, in the sense that the signals will have to pass through each intermediate átmiton to arrive to their destination, similarly to how a bishop can move through multiple cells in a single chess move (instantaneous), but only if each one of the intermediate cells on his path is unoccupied (causal).

In this section, some of the characteristics of átmita and their interactions will be discussed. For them to be able to form LANs and interact with each other, the following will be required axiomatically:

1. Each átmiton should be able to form connections with other átmita, similarly to the nodes of Fig. 1 and 2. The number of possible direct connections an átmiton can have (i.e., the number of its ports) will be defined axiomatically.

2. Each átmiton should have a unique address that permits it to exchange information (in the form of signals) with other átmita.

3. Each átmiton that has open ports (i.e., unused direct connections) should create and broadcast a signal containing the details of the open port, so that other átmita with corresponding open ports can move towards the broadcasting átmiton and jointly close their openings.

\subsubsection{Connections between átmita}

Two átmita that can exchange information directly, without it having to pass through any other átmiton, are said to have a direct connection. These átmita are nearest neighbors of each other. The number of these direct connections is given by the following Axiom:

\section{Axiom 1 - Number of direct connections:}

An átmiton can have a number of direct connections equal to twice the dimension of the Universe (two at each dimension, similarly to a simple cubic crystal).

In other words, an átmiton can have a maximum of $2 d$ nearest neighbors, where $d$ is the dimension of the Universe (i.e., a maximum of 6 nearest neighbors in 3D). With the nomenclature of Network theory, one could say that each attmiton has a number of $2 d$ ports, each one capable of forming one direct connection. Note that an átmiton can have less direct connections than the maximum permitted. This is of course to be expected, since any finite 3D simple cubic crystal should have open connections at least at its six surfaces, even if it is perfect inside (no holes, defects, etc).

More generally, two átmita, even if they do not have a direct connection, might be able to exchange information by passing signals from direct connection to the next. The connection distance, $d_{c}(A B)$, of those two átmita (denoted as $A$ and $B$ ) is given by the number of direct connections in the shortest continuous path that enables them to interact.

Evidently, the connection distance of two nearest neighbors defines the quantum of distance in this theory. Note that, by construction, a fraction of a mikos is nonsensical (i.e., $d_{c} \in \mathbb{N}$ ).

\subsubsection{Address of each átmiton}

Next, we turn to the notion of an átmiton's address, the equivalent of the IP address, that allows átmita to interact with each other. We will postulate axiomatically that the address of an átmiton should be unique.

Axiom 2 - Uniqueness of address:

Address of an átmiton is a set of integer numbers equal to the dimension of the Universe that acts as an identification for it. Each átmiton has its own frame of reference, in which it has the "zero" address, i.e., $(0,0,0)$ in 3 dimensions. Every átmiton translates all other addresses to its own frame. No two átmita can know to have the same address, when translated to the same frame of reference.

In other words, the átmiton's address is a locally defined property. Note that even though all átmita define their own address as being $(0,0,0)$, this does not contradict their uniqueness, as these addresses are in different frames. For example, in Fig. 3 , 
átmiton $\mathrm{B}$ is two miki to the right of átmiton $\mathrm{A}$, so, when translated into its own frame, all addresses are shifted by 2 miki to the left of what they are in the frame of reference of A. In this case, the transformation was straightforward, but in more complicated instances, the transformation can result in "wrongly" translated addresses. This will be the focus of a subsequent publication.

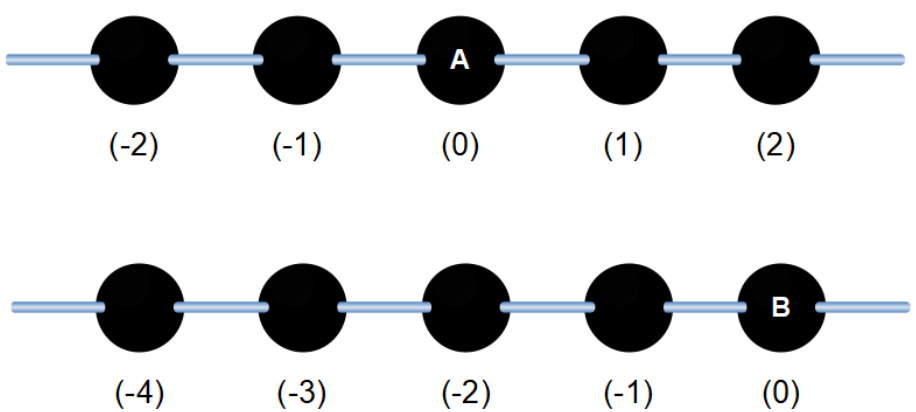

Figure 3. Image of an 1D array of átmita with their addresses in the frame of references of átmiton $\mathrm{A}($ top $)$ and $\mathrm{B}($ bottom).

The notion of an átmiton's address, leads to a second measure of distance between two átmita: The address distance, $d_{a}(A B)=d_{a}(\mathbf{p}, \mathbf{q})$ of two átmita $\mathrm{A}$ and $\mathrm{B}$ with addresses that correspond to the vectors $\mathbf{p}=\left(p_{1}, p_{2}, p_{3}, \ldots, p_{d}\right)$ and $\mathbf{q}=$ $\left(q_{1}, q_{2}, q_{3}, \ldots, q_{d}\right)$, where $d$ is the dimension of the Universe. This is given by the taxicab/rectilinear metric (i.e., the $L_{1}$ metric):

$$
d_{a}(\mathbf{p}, \mathbf{q})=\|\mathbf{p}-\mathbf{q}\|_{1}=\sum_{k=1}^{d}\left|p_{k}-q_{k}\right|
$$

\subsubsection{Signals}

Now we turn to the question of how some previously non-interacting átmita come together and form compound structures. We will require axiomatically that átmita with open ports (i.e., unused direct connections) would try to find others with relevant open ports, in order to converge with each other and close their corresponding openings. This is, in a sense, the root of all interactions in this theory. Here we will discuss some aspects of how these signals look and propagate on the network of átmita.

Axiom 3 - Signal generation and propagation:

An átmiton having (at least) an open port would create a signal broadcasting the open port, which would propagate on the network instantaneously by passing from direct connection to direct connection. Each such (instantaneous) move of the signal between neighboring átmita is called a step.

For instance, in $2 \mathrm{D}$, an átmiton that has an open port at the $[-\hat{x}]$ direction would create the (locally defined) signal $[-\hat{x}](0,0)$. Similarly to how each átmiton's address is translated in different frames of reference when passing from átmiton to átmiton, the same would happen to its signal. So if the above signal was sent by átmiton $A$ of Fig. 4, when it arrives at átmiton $B$ it will be translated to be $[-\hat{x}](-2,1)$, as point $A$ is 2 miki to the left and one mikos up of point $B$.

One main concept of átmiton theory is that all interactions arise from átmita responding to such signals, through a communication protocol. Even though this work will not cover all aspects of this protocol, we will postulate some basic processes that are absolutely necessary for the scope of this study.

\section{Protocol process 1 - Interaction:}

As part of the átmita's communication protocol, an átmiton receiving a signal which broadcasts an opening in the opposite direction from one of the receiving átmiton's open ports (e.g., signaling a $[-\hat{x}]$ opening to an átmiton having a $[+\hat{x}]$ open port) will interact with the incoming signal. As a result, it will try to close its corresponding open port by moving towards the átmiton broadcasting the signal. If the receiving átmiton does not have an open port in the opposite direction of the one broadcasted, then the signal will have no effect.

\subsubsection{LAN and particles}

It seems natural to bundle into a single entity all átmita that translate their addresses correctly from one local frame to all others'. This will be called a $L A N$, which is a set of átmita that share an effective frame of reference, meaning that for each pair of átmita $A$ and $B$ in the LAN, the connection distance equals the address distance (i.e., $\left.d_{c}(A B)=d_{a}(A B), \forall A, B \in L A N\right)$. We will call the largest LAN in the Universe Space. 


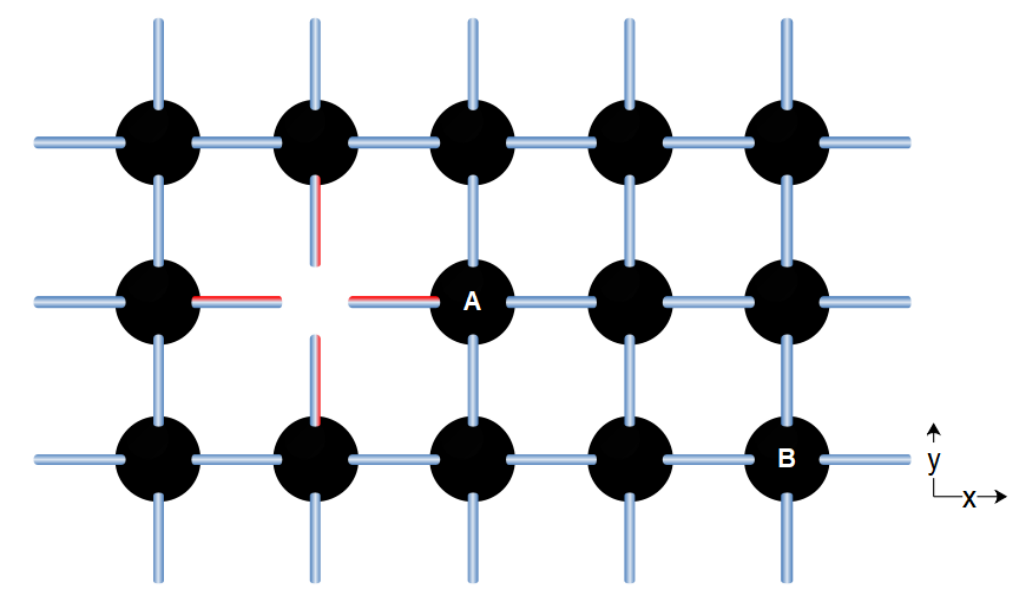

Figure 4. Figure of a $2 \mathrm{D}$ slab of átmita with an unoccupied position to the left of átmiton $\mathrm{A}$. This hole results in its four neighbors having an open port (red connections). Each of them will broadcast a signal with the details of the opening. All interactions in this theory are manifestations of such signals.

In the simple cases presented in Fig. 3 and 4, the connection distance coincides with the address distance, as in both cases all átmita shown are part of the same LAN. The case where there are two LANs with different effective frames of reference will be explored in detail in a subsequent publication.

When a LAN of átmita is placed inside another LAN, the former will be called internal and the latter substrate LAN. The only exception would be when we place a LAN directly in Space, in which case Space would act as a substrate, but usually it will not be denoted as such.

The átmiton of a substrate (or Space) on which a LAN is connected is called router átmiton. The router can be thought of being a part of both the LAN and its substrate, as it has the role of translating the addresses from one effective frame of reference to the other. The collection of LANs which are connected to Space through the same, single, router átmiton would then be called a particle. For example, the combination of the internal end external LANs (denoted collectively as LAN1) in Fig. 2, would be considered a particle.

\subsubsection{Time and motion}

So far we have discussed how individual átmita are connected and generate signals in a static manner. Next, the notions of time, movement and speed will be introduced.

Axiom 4 - Time stems from changes in the network's topology:

The internal topology and the position of each LAN (relative to other LANs) evolves in turns, each of which is defined as a quantum of time. It will be denoted as $T_{g}$ in natural units or $Q_{t}$ in SI and will be called a gyros (plural: gyroi) from the Greek word for the turn

Next, we will study how átmita can move on a lattice:

Protocol process 2 - hopping:

During each gyros, each átmiton can hop to fill a vacant port of an átmiton lying one mikos away.

The allowed (by Protocol 2) hop of one mikos per gyros of an átmiton moving in its LAN, or a LAN moving collectively on its substrate (by its router hopping on the substrate), defines the maximum allowed speed in this theory and will be denoted as the speed of light. A critical detail in Protocol 2 is that only movements to vacant ports on the lattice are allowed. This is a direct consequence of Axiom 2, as a movement to a filled position would result in two átmita sharing an address. Thus, in the situation depicted in Fig. 4, átmiton $A$ can hop to the vacant position on its left at the next gyros, but cannot go towards any other direction, as all of its other nearest neighbors' ports are filled. In addition, due to the same reason, átmiton $B$ of Fig. 4 will not move towards the vacant position, even if it has a relevant open port.

If the interaction between two átmita is intra-LAN, then each átmiton can move independently from the rest of its LAN towards the opening, as is the case for átmiton $A$ in Fig. 4. But if the interaction in extra-LAN, then the whole LAN should move in address-space towards the open port. Therefore, this motion of the LAN in address-space requires an actual motion of the particle's router in Space (or on its substrate more generally). 


\section{Particles in Space generate an effective curvature}

Since Space is a vast 3D network of átmita with the simple cubic topology without holes and only integer addresses are valid (Sect. 2), the LAN's router can be placed at neither an interstitial nor a substitutional position. Thus, the only way to place a LAN in Space would require to either increase the dimension of Space by 1 (from 3D to 4D, hence increase the number of possible direct connections for each átmiton by 2), or to (locally) re-define one of the three dimensions of the router átmiton to point towards its connection with the LAN and not towards its nearest neighbors at one dimension of Space (see Fig. 5).

Thus, to keep Space 3D, the router átmiton (denoted as $R$ in Fig. $5 b$ ) re-defines one of the $x$ - $y$ - or $z$-dimensions of its local coordinate system to coincide with the connection established with the LAN. Since one dimension of $R$ 's coordinate system is repurposed for connecting with the LAN, the two former connections of $R$ with the neighboring Space átmita across a dimension will have to break (see the red connections of Fig. 5b).

Which dimension chooses the router to repurpose, in order to connect with the LAN? According to Protocol 2 (see Sect. 2), the LAN connected to router $R$ can only hop to a neighboring átmiton of Space, if it has an open port. For example, for átmiton $G$ of Fig. 5 b to move in Space, its router $R$ has to hop by swapping positions with one of its neighbors in the $[-\hat{x}]$ or $[+\hat{x}]$ directions, as all other nearest neighbors of $R$ do not have open ports. Thus, the repurposed dimension is not fixed, rather is always parallel to the instantaneous motion of the particle in Space.

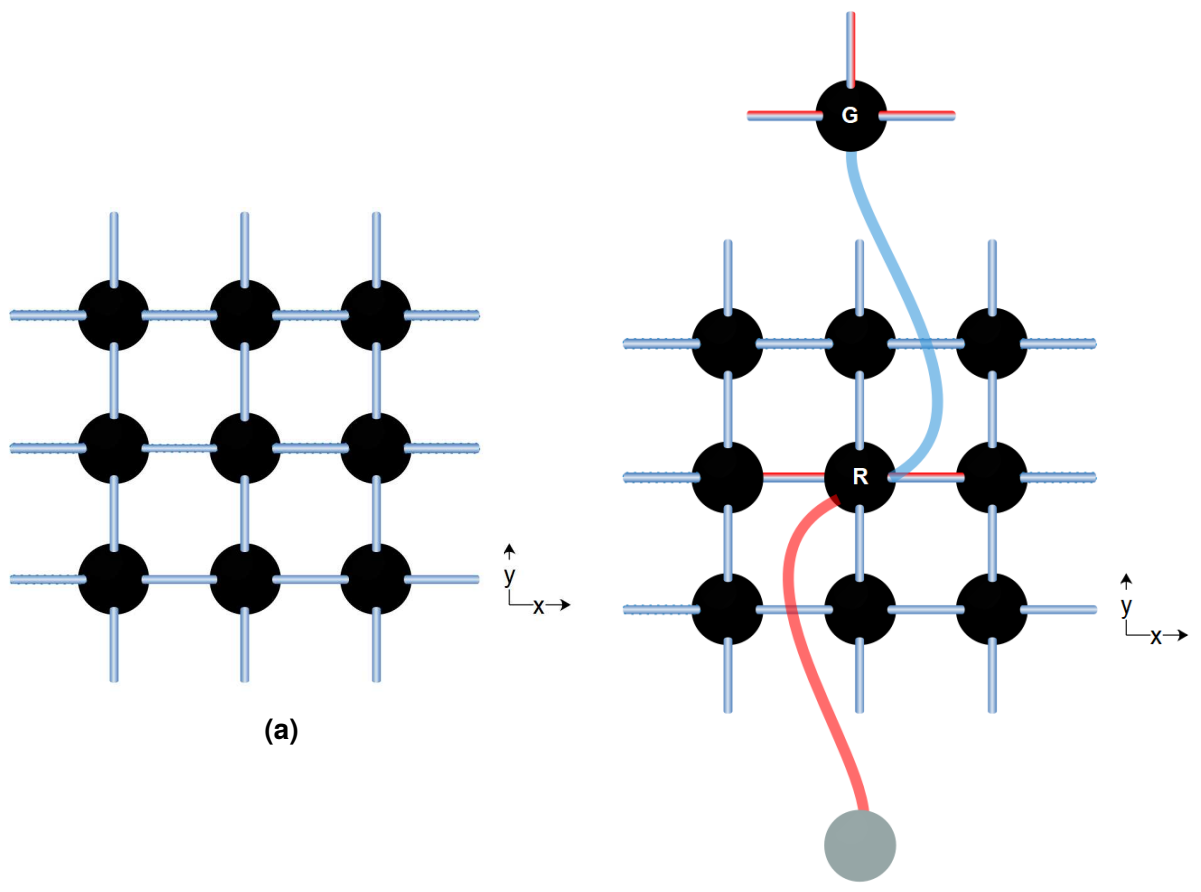

(b)

Figure 5. Map of connections between átmita in a portion of Space (shown in 2D) ( $a$ ) before and (b) after adding a LAN (consisting of a single átmiton, denoted as $G$ ) at the central position of the shown map, with the Space átmiton (denoted as $R$ ) acting as the router connecting the new LAN with Space. Red lines denote open connections and blue existing ones, while the gray circle emphasizes the fact that router $R$ has now an open port towards its local $[-\hat{y}]$ direction. The connection of the new LAN in the $y$-axis of router átmiton $R$ is fixed, whereas the fact that the broken Space bonds shown in this Figure are in the $x$-axis, indicates that during the next gyros the LAN can move either in the $[-\hat{x}]$ or $[+\hat{x}]$ directions by its router swapping positions with the corresponding neighboring Space átmiton.

The two nearest neighbors of the router in the dimension of the particle's instantaneous motion in Space have one broken connection each (Fig. 5b). Thus, the act of placing a LAN in Space changes the local geometry of Space, by increasing the distance of two previously neighboring points by 2 miki (see Sect. 2). Effectively, adding a particle to a point in Space increases the "curvature" of it, in the sense that it increases the connection distance $d_{c}$ between points in Space. This result is of course relevant to General Relativity's (GR) notion that energy (which macroscopically is proportional to the number of particles present at some volume) bends space-time by increasing the time it takes light to propagate between two given points in Space.

Massive objects, constantly generate due to their internal interactions copious amounts of new particles. The simplest and 
lowest energy ones (i.e., the gravitons) can be considered as nothing more than moving space impurities, which effectively make a given length seem longer at their presence. Microscopically, one can utilize a condensed matter picture and think mass as an impurity-generating part of the lattice of Space. These impurities will then diffuse away by means of a random walk ${ }^{10}$. Here it is suggested that the gravitational field is nothing more than the density distribution of these space-impurities.

Macroscopically, another way of viewing this effect is as a treadmill, in the sense that every particle moving in a gravitational field would have to cross the "extra space" introduced by space-impurities, in addition to the static background Space átmita. To understand how the treadmill effect and curvature can be related, consider the following analogy: In some airports there are some "conveyor belts" that allow for faster transport of pedestrians between terminals. If someone is walking at a constant speed - say $1 \mathrm{~ms}^{-1}$ - and walks on this belt opposite to its motion, they would cross a given distance - from point A to point B in a certain amount of time. If the same person walks beside the belt, on the regular sidewalk, with the same speed of $1 \mathrm{~ms}^{-1}$, they would go from point A to point B faster. Thus, the existence of the conveyor belt made the distance between two given points to look longer that what it would be in its absence, as the person had to transverse the "extra space" that the conveyor belt generates by its motion.

Although surveying the general equivalency of the space-impurity effect and GR's curvature of spacetime should be the focus of another publication, in Sect. 4 it is explicitly shown that the aforementioned effect in a discrete spacetime yields the Schwarzschild metric in the region outside a spherically-symmetric mass.

\section{Reproduction of the Schwarzschild metric based on the space-impurity diffusion model}

Let two inertial observers $A$ and $B$, standing still relative to each other and relative to a spherical mass $m$. $A$ is far away from $m$ (at "infinity"), whereas $B$ lies at a relatively small distance $r$ from it. There are no other massive objects close to them and observers $A$ and $B$ have an infinitesimal mass (compared to $m$ ), hence they are treated as test particles.

In this Section it will be shown that if $m$ generates a constant flux of space-impurities, which diffuse away from it by means of a random walk, then the spacetime metric at the position of observer $B$ is given by the familiar Schwarzschild line element.

\subsection{Diffusion of space-impurities generated by $m$}

To calculate the density profile of the space-impurities around $m$, let us split space in infinitesimal shells of thickness $d r$, each centered around a constant radius from the source $m$. It is evident from the spherical symmetry of the problem, that the density inside each such shell will be everywhere equal.

If somehow $m$ was abruptly materialized at its position at some instance in time, then the density of space-impurities in each shell would be zero and the flux of new impurities generated at the innermost infinitesimal shell would be diffusing away from $m$ at a rapid pace, due to the big concentration gradient between the innermost and all other shells. Gradually, the concentration gradient close to $m$ would approach a steady-state situation, in which the outflux and influx of impurities in each shell would be equal. Here we will calculate the concentration of impurities at the shell in which observer $B$ lies (at a radius $r$ from $m$ ), assuming this steady-state situation.

After shell $r$ has reached its steady-state, the concentration of impurities - denoted as $c_{i m}(r)-$ will henceforth have a zero Laplacian $^{11}$ (Eq. 2):

$$
\nabla^{2} c_{i m}(r)=0
$$

Since we are in a situation of spherical symmetry, Eq. 2 can be written as:

$$
\frac{1}{r^{2}} \frac{\partial}{\partial r}\left(r^{2} \frac{\partial c_{i m}(r)}{\partial r}\right)=0
$$

After some algebra, Eq. 3 leads to the following second-order differential equation:

$$
\frac{\partial^{2} c_{i m}(r)}{\partial r^{2}}+\frac{2}{r} \frac{\partial c_{i m}(r)}{\partial r}=0
$$

which has the solution: $c_{i m}(r)=\frac{B}{r}+C$, with $B, C$ constants. Here we will set $C=0$, as it is irrelevant to the dynamics in question. Also, the flux of new impurities that a given mass $m$ generates should be proportional to $m$ (Sect. 3), which would mean in turn that $B \propto m$. We will thus set $B=A \cdot m$. Then, $c_{i m}(r)$ is given by:

$$
c_{i m}(r)=\frac{A \cdot m}{r}
$$


The final result of Eq. 5 notably has the same form as the gravitational potential: $V(r)=\frac{G \cdot M}{r}$. Note that (by dimensional analysis) $A$ has units of $\left[M^{-1} L^{-2}\right]$, so we can relate $A$ to $G$ by the formula:

$$
A \cdot Q_{L}^{3}=G / c^{2}
$$

where $Q_{L}$ is the quantum of distance in SI units (see Sect. 2).

In the next section, these results are used to recover the Schwarzschild metric.

\subsection{The spacetime metric at a distance $r$ from the source of impurities}

Let observers $A$ and $B$ be measuring lengths using rigid rulers and time by measuring how long light takes to cross a given length. If $m$ was absent, then when $A$ had measured a time interval $\Delta t, B$ would have measured an interval $\Delta \tau=\Delta t$, which would correspond to the time interval that takes light to cross some length $L$ (i.e. $L=\Delta t \cdot c$ ).

Now let the mass $m$ be present near $B$. Here, the length of $L$ will be considered negligible $(L<<r)$, so the tidal effects due to different values of $c_{i m}(r)$ at different parts of $L$ can be neglected. As $A$ is far away, we can neglect the effects of $m$ on the way $A$ measures time and length, so the interval $\Delta t$ is unchanged. But the watch of observer $B$ will be running slower, as its light beam will now have to cross some extra space átmita, due to the impurities produced by $m$, in addition to the fixed átmita of length $L$ (i.e., the ones that make up $L$ in the absence of space-impurities).

Each such impurity increases the distance between two points in Space (lying at opposite sides of it) by 2 units of length (i.e., two miki, see Sect. 3). If we denote the quantum of distance as $Q_{L}$ (in SI units), then a given length $L$, lying at a distance $r$ from $m$ would increase by:

$$
\Delta L=L \cdot c_{i m}(r) \cdot Q_{L}^{2} \cdot 2 Q_{L}
$$

The factor $Q_{L}^{2}$ is added to Eq. 7 in order to turn $c_{i m}$ from units of [\# of impurities]/[unit volume] to [\# of impurities]/[unit length].

Nonetheless, observer $B$ would still consider the length of its rigid ruler, $L$, to be fixed. Thus, during the same time interval $\Delta t$, as measured by observer $A, B$ 's watch would measure an interval of:

$$
\begin{aligned}
\Delta \tau^{\prime} & =(L-\Delta L) / c \\
& =L / c-(L / c) c_{i m}(r) 2 Q_{L}^{3} \\
& =\Delta t\left[1-\left(2 Q_{L}^{3} A m\right) / r\right] \\
& =\Delta t\left(1-r_{s} / r\right)
\end{aligned}
$$

where we set $r_{s}=2 Q_{L}^{3} A m$, in order to connect the microscopic treatment of this study with the macroscopically-defined Schwarzschild radius. Note that, in GR: $r_{s}=2 G m / c^{2}$, thus:

$$
\begin{aligned}
& 2 Q_{L}^{3} A m=2 G m / c^{2} \\
& A Q_{L}^{3}=G / c^{2}
\end{aligned}
$$

The last line of Eq. 9 independently reproduces Eq. 6.

Next, we allow the time interval $\Delta t$ to become infinitesimal, multiply both sides of Eq. 8 by $c$ and we drop the accent from $d \tau^{\prime}$. Squaring both sides of Eq. 8, then yields:

$$
(c d \tau)^{2}=(c d t)^{2}\left(1-r_{s} / r\right)^{2}
$$

Finally, to construct the metric, we note that due to the spherical symmetry of the problem, the angular part of the metric should be the familiar Minkowski one in spherical coordinates. The radial component of the metric, as measured by observer $B$, is then:

$$
\begin{aligned}
& c^{2} d \tau^{2}=d r^{2} \\
& \left(1-r_{s} / r\right)^{2} c^{2} d t^{2}=d r^{2} \\
& \left(1-r_{s} / r\right) c^{2} d t^{2}=\left(1-r_{s} / r\right)^{-1} d r^{2}
\end{aligned}
$$


Adding the familiar angular part, one recovers the Schwarzschild metric, here with the sign convention $(-,+,+,+)$ :

$$
d s^{2}=-\left(1-r_{s} / r\right) c^{2} d t^{2}+\left(1-r_{s} / r\right)^{-1} d r^{2}+r^{2} d \theta^{2}+r^{2} \sin ^{2} \theta d \phi
$$

\section{Conclusions and future work}

In conclusion, this study presented some fundamental properties of a type of communication node - called "átmiton" - and of the protocol that allows such nodes to interact. It was suggested that systems of átmita, called "LANs", posses the equivalent of certain quantum properties, such as seeming point-like to outside observers, while having an internal field-like structure. It was suggested that it this context, space might be considered as a lattice of atmita with the simple cubic crystal structure and that the position of each particle/LAN might correspond to the position of its "router" átmiton in Space. The router connects the LAN with other LANs through Space and allows the exchange of signals between them.

It was postulated that signals are generated when an átmiton has unused connections (i.e., open ports) and that these signals would cause other átmita with relevant open ports to move towards the broadcasting átmiton and annihilate their relevant openings. To be able to send this information, each átmiton should have a unique address. Since that address is locally defined, the router átmiton of the LAN should translate it to some form that can propagate in Space, without address-collisions, similarly to how a telecommunication router translates the private IP of a computer to a public IP address, capable of propagating on the Internet.

It is arguable that the long-range physical interactions might arise from fundamental signal exchanges between átmita belonging in different LANs, with their signals translated by their router átmita and propagated through Space. In contrast, the short-range forces might arise when two LANs come close enough to connect directly - not through Space - by forming a unifying LAN. Then, their signals won't have to be translated by their routers, thus these forces will probably have distinct characteristics and symmetries. In any case, further advances are needed in the details of the communication protocol in order to be able to derive the specific characteristics of those forces.

In this publication, it was shown that the only way to turn an átmiton of Space into the LAN's router, without increasing the dimensionality of Space, is by locally deform Space's structure. This lattice defect increases the connection distance of two nearest neighbors Space átmita by two units, thus increases the effective distance of any two given points in Space, that lie at opposite sides of the deformity.

It stands to reason that the larger the mass of a macroscopic body, the more such defects will be produced per unit time by its internal interactions. These defects will then be diffusing in all directions of the lattice of Space by a random walk. It was suggested that the density of these defects is equivalent - or at least proportional - to the general relativity's curvature of spacetime. This equivalence was explicitly shown for the specific case of a spherically symmetric body. In the vicinity of such a mass, Schwarzschild metric was recovered using the aforementioned diffusion-driven impurity model. Even though in this paper the discussion was in the context of Átmiton theory, this result can be argued to be true in a more general setting: The sole requirements are for space to be discrete (i.e., like a lattice) and for each particle to deform Space locally, acting as an impurity.

Moving on with this study, another publication should try to address whether the impurity-driven mechanism presented here is equivalent to GR's curvature in general. Note that although GR is not linear, the equivalence of the two models for the specific case of the spherical mass suggests that they agree with each other at first order, since any mass can be though as being made of many infinitesimal spherical masses.

The next step in the development of Átmiton theory is to consider what addresses the átmita of the added LAN can occupy, without any of them colliding with addresses already claimed by Space átmita. This is not straightforward, as Space already occupies a vast volume in address-space and interstitial positions (i.e., non-integer addresses) are not valid.

Nonetheless, to be able to discuss the aforementioned problems, one needs first to survey other aspects and processes of átmita's communication protocol. Subsequent publications will expand the scope of this construct by gradually answering several questions that this study left open. For example, here it was suggested that spin might naturally arise in the context of Átmiton theory, if we place an internal LAN inside another LAN. This suggests that most - if not all - particles of the Standard Model might have second-order (i.e., embedded) structures, like a Russian doll. It is thus important to construct all possible second-order structures and try to understand each structure's characteristics, with the ultimate intention to correlate each one with a known particle of the Standard Model.

\section{Acknowledgments}

The author received financial support for part of this study from the IsoSiM fellowship. The author would like to acknowledge Yannis Hadjichristos for his valuable remarks during the early stages of this theory's development. 


\section{References}

1. Mehra, J. \& Rechenberg, H. The Historical Development of Quantum Theory (Springer-Verlag New York, 1982).

2. Bohm, D. A suggested interpretation of the quantum theory in terms of "hidden" variables. i. Phys. Rev. 85, 166-179, DOI: 10.1103/PhysRev.85.166 (1952).

3. Dewitt, B. S. \& Graham, N. The Many Worlds Interpretation of Quantum Mechanics (Princeton Legacy Library, 2016).

4. Danielsson, U. Introduction to string theory. Reports on Prog. Phys. 64, 51-96, DOI: 10.1088/0034-4885/64/1/202 (2000).

5. Rovelli, C. Loop quantum gravity. Living Rev. Relativ. 11, DOI: 10.12942/lrr-2008-5 (2008).

6. Einstein, A., Podolsky, B. \& Rosen, N. Can quantum-mechanical description of physical reality be considered complete? Phys. Rev. 47, 777-780, DOI: 10.1103/PhysRev.47.777 (1935).

7. Bell, J. S. On the einstein podolsky rosen paradox. Phys. Physique Fizika 1, 195-200, DOI: 10.1103/PhysicsPhysiqueFizika. 1.195 (1964).

8. Einstein, A. Die grundlage der allgemeinen relativitätstheorie (the foundations of the general theory of relativity). Annalen der Physik 354, 769-822, DOI: 10.1002/andp.19163540702 (1916). https://onlinelibrary.wiley.com/doi/pdf/10.1002/andp. 19163540702.

9. Schwarzschild, K. On the Gravitational Field of a Mass Point According to Einstein's Theory. Abh. Konigl. Preuss. Akad. Wissenschaften Jahre 1906,92, Berlin,1907 1916, 189-196 (1916).

10. Chandrasekhar, S. Stochastic problems in physics and astronomy. Rev. Mod. Phys. 15, 1-89, DOI: 10.1103/RevModPhys.15.1 (1943).

11. Mehrer, H. Diffusion in solids. Springer series in solid state sciences (Springer, 2007). 


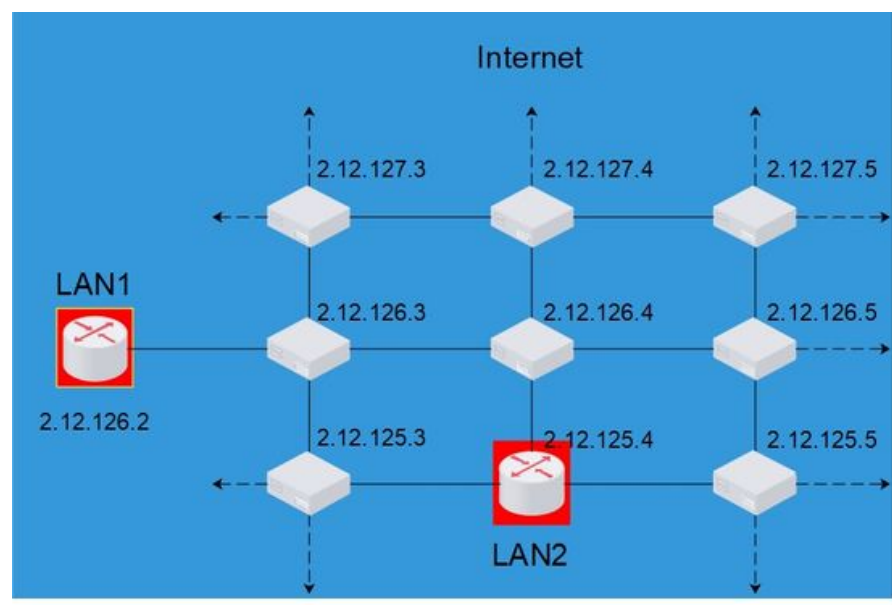

(a)

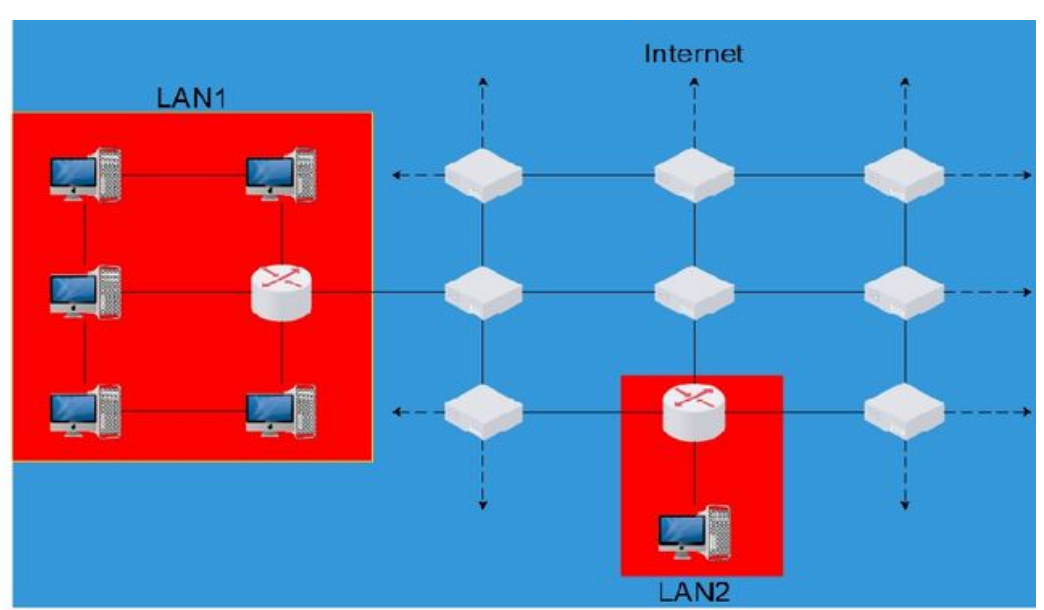

(b)

\section{Figure 1}

Image of two simple LANs (in red), each with a different number of internal nodes (computers), connected to the Internet (in blue) through a router. (a) Map of the public IP addresses. In this view, each LAN seems point-like, as it has one direct connection to the Internet and a single public IP address. (b) The map of all connections between nodes on the same network. Note that in telecommunication networks, the IP addresses of neighboring nodes are generally independent of each other, not in a series as depicted here.

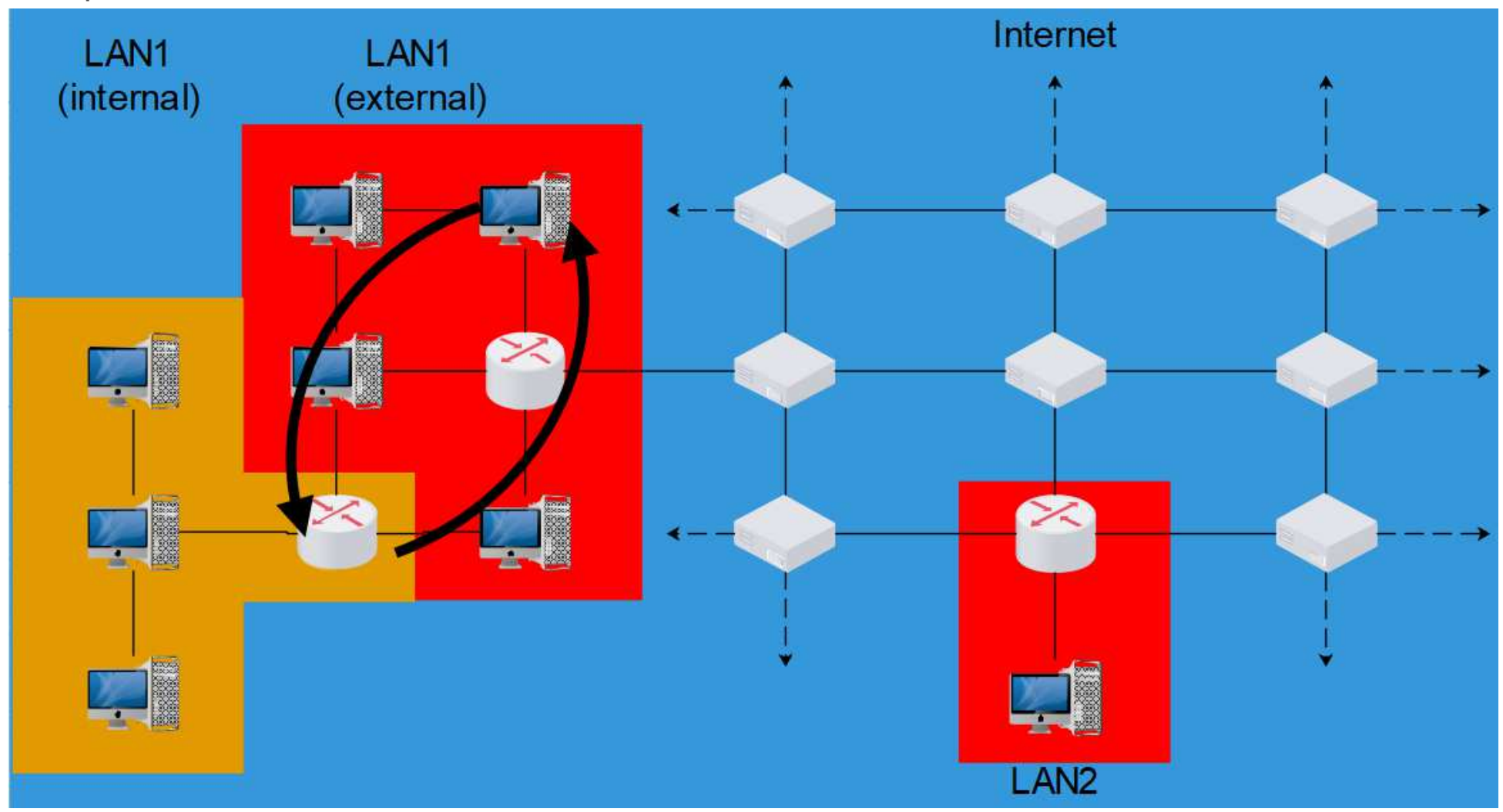

Figure 2 
Image of an internal LAN, placed inside LAN1, with its router's position on the (external) LAN1 shifting counter-clockwise. Under appropriate definitions, such a motion can be associated with intrinsic angular momentum, even though the whole LAN1 seems still point-like, from the point of view of an outside observer (see Fig. 1a).
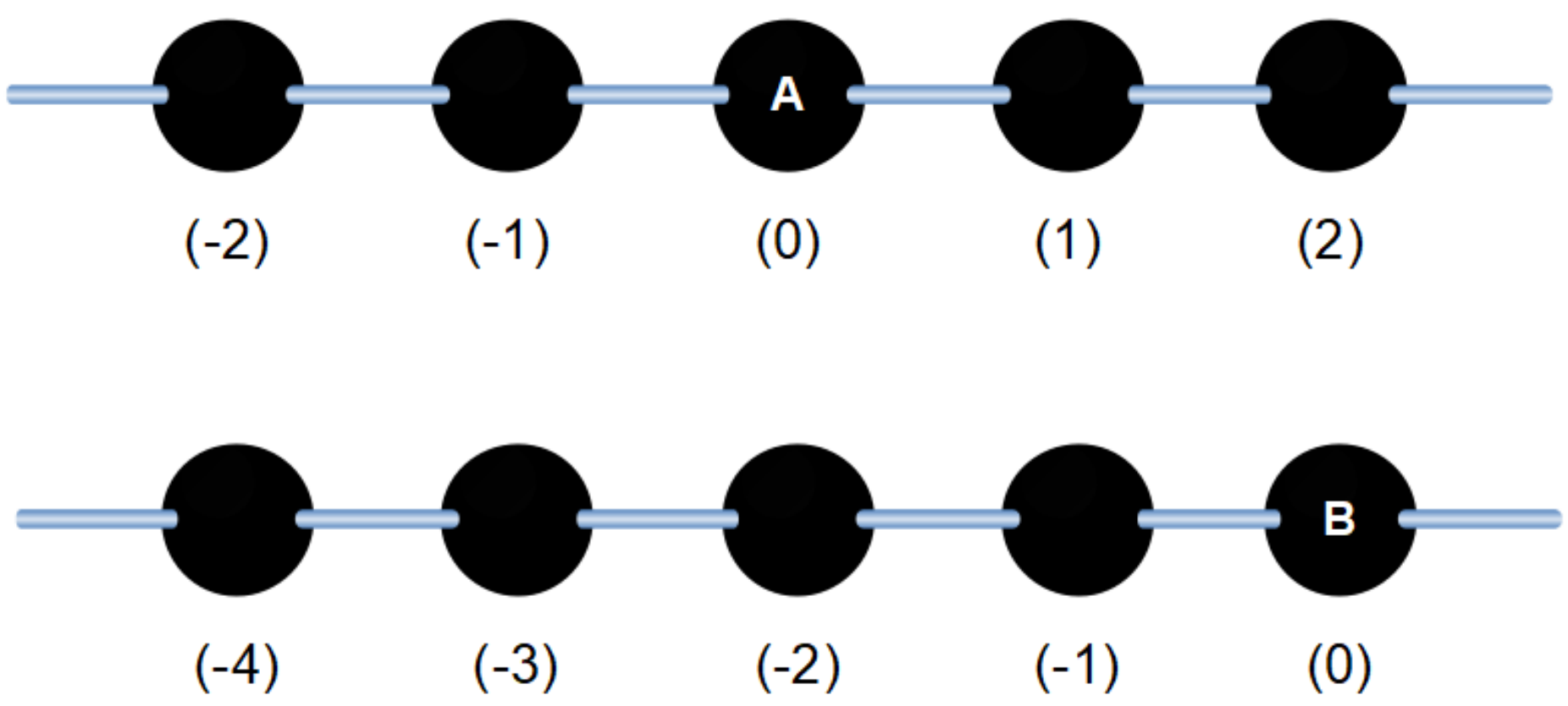

Figure 3

Image of an 1D array of átmita with their addresses in the frame of references of átmiton A (top) and B (bottom). 


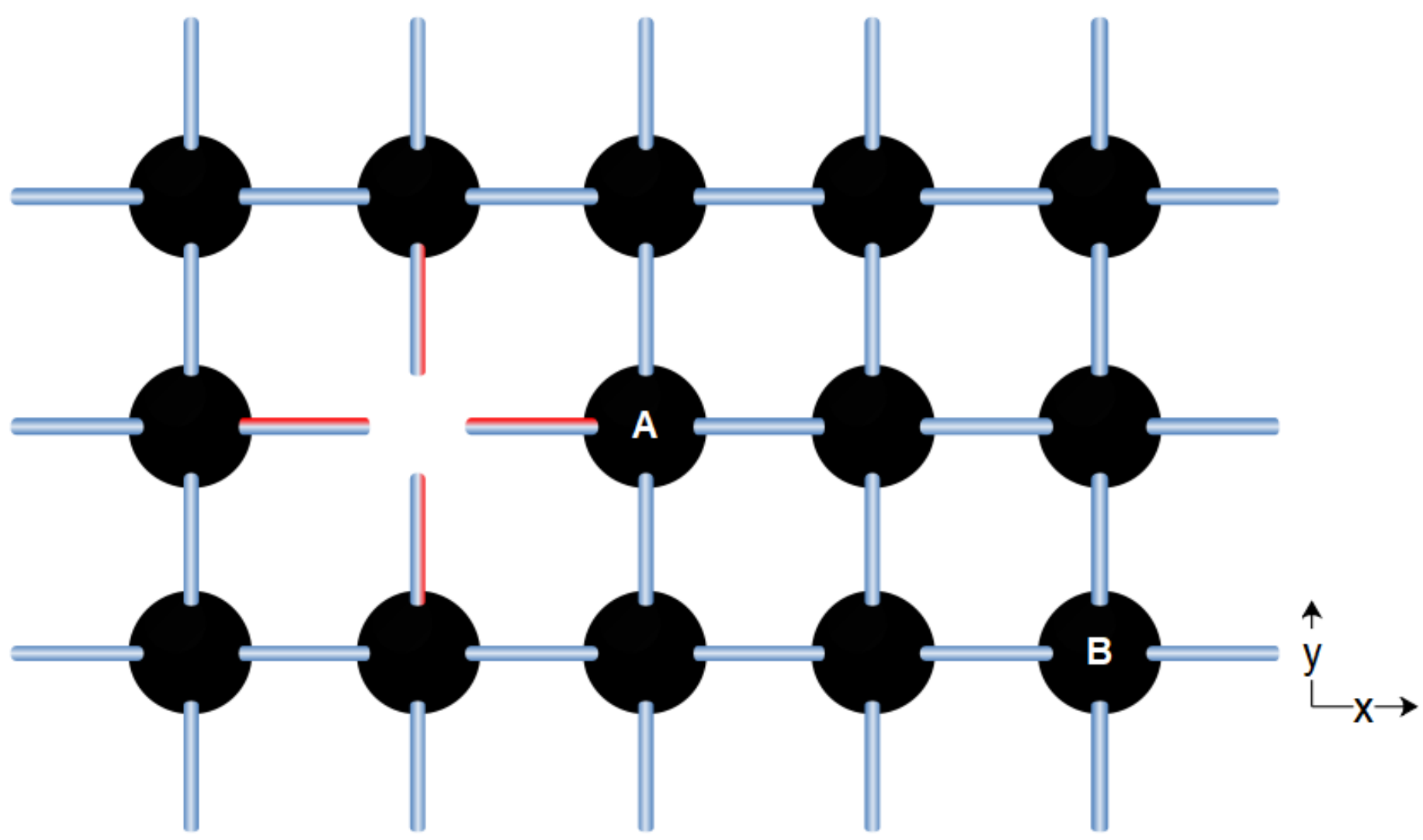

Figure 4

Figure of a 2D slab of átmita with an unoccupied position to the left of átmiton $A$. This hole results in its four neighbors having an open port (red connections). Each of them will broadcast a signal with the details of the opening. All interactions in this theory are manifestations of such signals. 

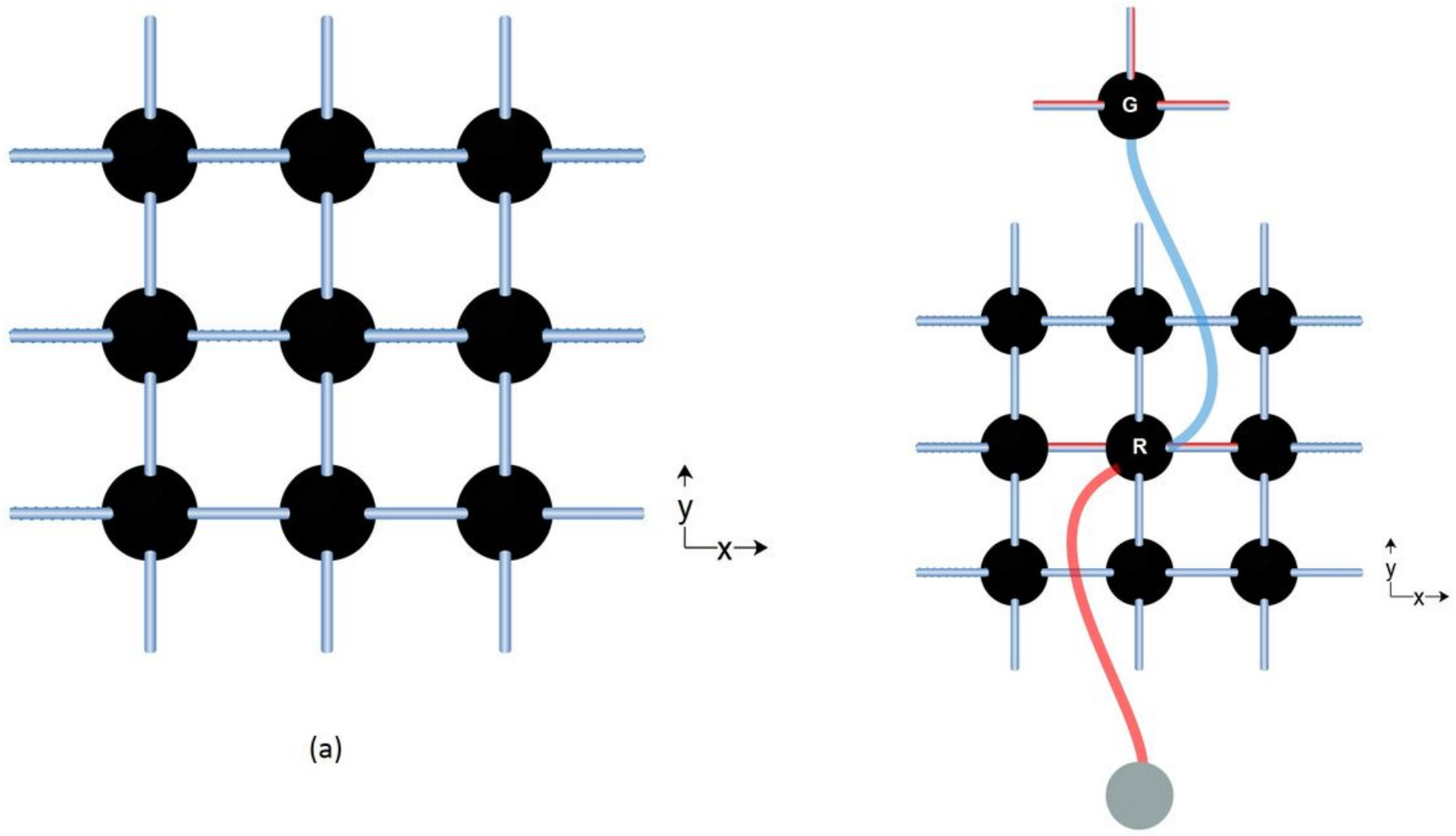

(a)

(b)

Figure 5

Please see the Manuscript PDF file for the complete figure caption. 